\title{
Interesting Examples of Violation of the Classical Equivalence Principle but Not of the Weak One
}

\author{
Antonio Accioly (D) and Wallace Herdy (D) \\ Coordenação de Cosmologia, Astrofísica e Interações Fundamentais (COSMO), Centro Brasileiro de Pesquisas Físicas (CBPF), \\ Rua Dr. Xavier Sigaud 150, Urca, 22290-180 Rio de Janeiro, RJ, Brazil
}

Correspondence should be addressed to Antonio Accioly; accioly@cbpf.br

Received 4 December 2017; Revised 20 April 2018; Accepted 3 June 2018; Published 8 July 2018

Academic Editor: Edward Sarkisyan-Grinbaum

Copyright (C) 2018 Antonio Accioly and Wallace Herdy. This is an open access article distributed under the Creative Commons Attribution License, which permits unrestricted use, distribution, and reproduction in any medium, provided the original work is properly cited. The publication of this article was funded by SCOAP ${ }^{3}$.

\begin{abstract}
The equivalence principle (EP) and Schiff's conjecture are discussed en passant, and the connection between the EP and quantum mechanics is then briefly analyzed. Two semiclassical violations of the classical equivalence principle (CEP) but not of the weak one (WEP), i.e., Greenberger gravitational Bohr atom and the tree-level scattering of different quantum particles by an external weak higher-order gravitational field, are thoroughly investigated afterwards. Next, two quantum examples of systems that agree with the WEP but not with the CEP, namely, COW experiment and free fall in a constant gravitational field of a massive object described by its wave-function $\Psi$, are discussed in detail. Keeping in mind that, among the four examples focused on in this work only COW experiment is based on an experimental test, some important details related to it are presented as well.
\end{abstract}

\section{Introduction}

The equivalence principle (EP) is intrinsically connected to the history of gravitation theory and has played an important role in its development. Newton regarded this principle as such a cornerstone of mechanics that he devoted the opening paragraph of the Principia to it.

Before discussing some important aspects of the EP, we would like to explain the two different meanings usually attributed within the context of physics to the words nonlocal and local.

A nonlocal principle is valid everywhere in space and time (space-time) as far as nonrelativistic (relativistic) theories are concerned, while a local principle is restricted only to one point of space and time (space-time).

On the other hand, the so-called principle of locality states that an object is only directly influenced by its immediate surrounding. A theory which includes the principle of locality is said to be a local theory. Locality evolved out of field theories of classical physics. The concept is that, for an action at one point to have an influence at another point, something in the space between those points such as a field must mediate the action. To exert an influence, something, such as a wave or particle, must travel through the space between the two points, carrying the influence. It is worthy of note that the special theory of relativity limits the speed at which all such influences can travel to the speed of light $c$. As a consequence, the principle of locality allows us to conclude that an event at one point cannot cause a simultaneous result at another point. In other words, an event at point $A$ cannot cause a result at point $B$ in a time less than $T=D / c$, where $D$ is the distance between the points.

In 1935, Einstein, Podolsky, and Rosen in their EPR paradox theorized that quantum mechanics might not be a local theory since a measurement made on a pair of separated but entangled particles causes a simultaneous effect, the collapse of the wave-function, in the remote particle (i.e., an effect exceeding the speed of light). But because of the probabilistic nature of wave-function collapse, this violation of locality cannot be used to transmit information faster than light. In 1964 Bell formulated the "Bell inequality", which if violated in actual experiments, implies that quantum mechanics violates either locality or realism, another principle which relates to the value of unmeasured quantities. The two principles are commonly referred to as a single principle, local realism. 
Experimental tests of the Bell inequality show that quantum mechanics seem to violate either locality or realism. Nevertheless, critics have noted that these experiments contained "loopholes", which prevented definite answers to this question. This might now be resolved: in 2015 Hanson at Delft University performed what has been called the first loophole free experiment [1].

In summary, the concepts of local and nonlocal principles are totally different from those concerning principles of locality and nonlocality. They cannot be used as synonymous.

We are now ready to examine, in passing, some relevant feature related to the EP in the framework of both Newton and Einstein gravity.

To begin with we discuss the notion of the EP in the context of Newtonian theory.

In the framework of Newton's theory we have two equivalence principles: Galileo's equivalence principle and Newton's equivalence principle. The Galileo's equivalence principle states that, in a given gravitational field, all test particles, of the same initial velocity, fall with the same acceleration. The requirement that all particles must be put at the given point with the same initial velocity is necessary because some pseudo-forces, such as the Coriolis force, are velocity-dependent. On the other hand, Newton's equivalence principle asserts that the gravitational mass of any system equals its inertial mass, or, in more succint terms, $m_{\text {inertial }}=$ $m_{\text {gravitational }}$. Note that the Galileo and the Newton principles should be regarded as complementary. Indeed, the former is a statement on the behavior of a special class of system (test particles) placed in arbitrary weak gravitational fields, while the latter is a statement on the behavior of an arbitrary system put in a weak gravitational field. These two principles have a nonlocal character; i.e., they are valid everywhere in space and time [2-5]. From now on we shall designate Newton's equivalence principle as the classical equivalence principle (CEP) of Newtonian theory. Once again we call attention to the fact that the CEP is a nonlocal principle; i.e., it is valid everywhere in space and time.

As far as Einstein gravity is concerned, two EP are generally contemplated: the weak equivalence principle (WEP) and the Einstein one (EEP). The WEP asserts that locally we cannot distinguish between inertial and gravitational fields through 'falling body experiments'. Since the WEP and the CEP are locally identical, the difficulty, at the first sight, of differentiating them in an easy way increases. Consequently, some researchers are led to the common misconception that they coincide even nonlocally (see for instance [6-10]).

Undoubtedly, the two great pillars of modern physics are quantum mechanics and general relativity. Nevertheless, the merging of these theories has not been accomplished despite the herculean efforts employed by so many distinguished physicists. What is the rationale for such an incompatibility between these outstanding theories? Perhaps it is the conceptual difficulty of reconciling the local character of the WEP with the nonlocal character of the uncertainty principle.

We call attention to the fact that the validity of both general relativity and WEP can be characterized by the PPN parameter $\gamma$, which reflects the level of space curvature per unit rest mass (for a recent review see [3]). As is well known, $\gamma=1$ as far as general relativity is concerned. On the other hand, the measurement of the absolute value of $\gamma$ has reached high accuracy in Astronomy. In fact, Krauss and Tremaine [11], as well as Longo [12], by observing a neutrino burst within $3 \mathrm{~h}$ of the associate optical burst from supernova 1987A in the Large Magellanic Cloud, were able to devise a new test of the WEP, by demonstrating that neutrinos and photons follow the same trajectory in the gravitational field of the galaxy. Recently, Wei et al. [13] probed WEP with fast ray bursts, while $\mathrm{Yu}, \mathrm{Xi}$, and Wang [14] found a robust method to test the WEP. More recently Yu and Wang [15] tested WEP with strongly lensed cosmic transients. It is worth noticing that every test of general relativity is a potentially deadly test or a possible probe for new physics. It is amazing that this theory, which came to light 100 years ago out of almost pure thought, has managed to survive every test. Of course, the possibility of finding a discrepancy will continue to drive experiments for years to come and these experiments will search for new physics beyond Einstein at many different scales: the large distance scales of the astrophysical, galactic, and cosmological realms; scales of very short distances or high energy; and scales related to strong or dynamical gravity [3].

EEP, on the other hand, embodies WEP, local Lorentz invariance-the outcome of any local non-gravitational experiment is independent of the velocity of the freely falling reference frame in which it is performed-and local position invariance - the outcome of any local non-gravitational experiment is independent of where and when it is performed [3]. EEP may be considered in the broadest sense of the term as the heart and soul of gravity theory. It would not be an exaggeration to say that if the EEP holds, then gravitation must necessarily be a 'curved space-time' phenomenon; in other words, the effects of gravity must be equivalent to the effects of living in a curved space-time [3]. Around 1960, Schiff conjectured that any complete and self consistent theory of gravity that obeys the WEP must also, unavoidably, obey the EEP [16]. This surmise is known as Schiff's conjecture. According to it the validity of the WEP alone should guarantee the validity of the local Lorentz and position invariance, and thus of the EEP. However, a rigorous proof of Schiff's conjecture is improbable. In fact, some special counterexamples are available in the literature [17-20]. Nevertheless, there are some powerful arguments of 'plausibility', such as the assumption of energy conservation [21] and the THє $\mu$ formalism [22], among others, that can be formulated.

In the seminal work by Will [3] entitled "The Confrontation between General Relativity and Experiment" it is asserted that EEP is well supported by experiments such as the Eötvos experiment, tests of local invariance, and clock experiments. In the aforementioned work, ongoing tests of EEP and the inverse square law with the aim of searching for new interactions arising from unification or quantum gravity are also discussed. Actually, EEP and related tests are currently viewed as ways to discover or place constraints on new physical interactions, or as a branch of "non-accelerator particle physics", searching for the possible imprints of highenergy particle effects in the low-energy realm of gravity. On 
the phenomenological side, the idea of using EEP tests in this way has its origin in the middle 1980s, with the search for a "fifth" force. In 1986 Fischbach et al. suggested the existence of a fifth force in nature [23]. Nevertheless, the physical community came to the conclusion that there was no credible evidence for a fifth force of nature of a type and range proposed by Fischbach et al. (for a review see, for instance, [24-26]). As far as short-range modifications of Newtonian gravity are concerned, no deviations from the inverse square law have been found up to now at distances between tens of nanometers and $10 \mathrm{~mm}$ [27-29].

A natural question must now be posed: what is the connection between the EP and quantum mechanics? As is well known, quantum tests of the EP are radically different from the classical ones because classical and quantum descriptions of motion are fundamentally unlike. In particular, the universality of free fall (UFF) possesses a clear significance in the classical context. Now, how both UFF and WEP are to be understood in quantum mechanics is a much more subtle point. It is generally implicitly assumed that quantum mechanics is valid in the freely falling frame associated with classical test bodies. Nonetheless, an unavoidable problem regarding quantum objects is the existence of half integer spins, which have no classical counterpart. For integer spin particles, the EP can be accounted for by a minimal coupling principle (see Sections A.1 and A.3 of Appendix A), while the procedure to couple a spin $1 / 2$ field to gravity is much more complex and requires the use of a spinorial representation of the Lorentz group (see Section A.2 of Appendix A).

On the other hand, the most cited scientific experiment claimed to support the idea that, at least in some cases, quantum mechanics and the WEP can be reconciled, that is COW experiment [7]. Although this test, as we shall prove, is in accordance with the WEP, it is in disagreement with the CEP. Another example of a possible quantum mechanical violation of the CEP but not of the WEP is provided by analyzing free fall in a constant gravitational field of a massive object described by its wave-function $\Psi$.

At the semiclassical level an interesting event in which the CEP is also supposed to be violated but not the WEP is the tree-level deflection of different quantum particles by an external weak higher-order gravitational field. We recall beforehand that in Einstein theory the scattering of any particle by an external weak gravitational field is nondispersive which, of course, is in agreement with the WEP. In other words, the deflection angle of all massive particles will be exactly equal. The same is valid for the massless particles. Obviously, the deflection angle will be different whether the particle is massive or massless. A crucial question must then be posed: why to study at the tree level the bending of quantum particles in the framework of higherderivative gravity? It is not difficult to answer this question. Higher-derivative gravity is the only model that is known to be renormalizable along its matter couplings up to now [30]. Nonetheless, since this system is renormalizable, it is compulsorily nonunitary [31, 32]. We call attention to the fact that the breaking down of unitarity is indeed a serious problem. Fortunately, we shall only deal with the linearized version of higher-derivative gravity, which is stable [33]. The reason why it does not explode is because the ghost cannot accelerate owing to energy conservation. Another way of seeing this is by analyzing the free-wave solutions. We remark that this model is not in disagreement with the result found by Sotiriou and Faraoni [34]. In fact, despite containing a massive spin-2 ghost, as asserted by these authors, the alluded ghost cannot cause trouble [35]. Another probable example at the tree level of violation of the CEP but not of the WEP is provided by Greenberger gravitational Bohr atom [6].

Our main goal here is to explicitly show that, in all situations described above, the WEP is not violated but the CEP is.

The article is organized as follows.

In Section 2 we study the following semiclassical examples:

(i) Greenberger gravitational Bohr atom.

(ii) Tree-level scattering of different quantum particles by an external weak higher-order gravitational field.

After a careful investigation of both models, we came to the conclusion that they do not violate at all the WEP but are not in accordance with the CEP. As far as the second example is concerned, it is worthy of note that the resulting deflection angles are dependent on both spin and energy. In addition the well-known deflection angles (related to both massive and massless particles) predicted by general relativity are recovered through a suitable limit process.

In Section 3 we analyze two quantum examples: COW experiment and free fall in a constant gravitational field of a massive object described in quantum mechanics by the wavefunction $\Psi$. Again, these systems are in accordance with the WEP but not with the CEP.

Our comments are presented in Section 4.

The lengthy calculations concerning the computation of unpolarized cross sections for the scattering of different quantum particles by an external weak higher-order gravitational field are put in Appendix A.

We use natural units throughout and our Minkowski metric is diag $(1,-1,-1,-1)$.

\section{Two Examples of Semiclassical Violation of the CEP but Not of the WEP}

We analyze in the following two examples of semiclassical violation of the CEP but not of the WEP in a gravitational field.

2.1. Greenberger Gravitational Bohr Atom. As far as we know, Greenberger [6] was the first to foresee the existence of massdependent interference effects related to a particle bound in an external gravitational field.

Here we are particularly interested in analyzing Greenberger gravitational Bohr atom, which from the classical point of view consists of a small mass $m$ bound to a very much larger mass $M$ by the potential $V(r)=-G M m / r$, in the limit where all recoil effects may be neglected. If we restrict ourselves to circular orbits, we arrive at the conclusion that classically $\omega^{2} r^{3}=G M$ (see Figure 1). 


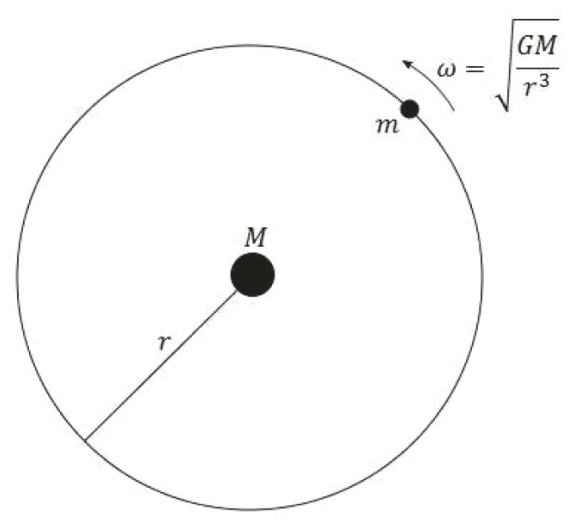

FIGURE 1: Greenberger classical gravitational Bohr atom with circular orbit.

From this point on Greenberger applied the same postulate proposed by Bohr.

'The particles move in orbits restricted by the requirement that the angular momentum be an integer multiple of $\hbar$. Therefore, according to this postulate for circular orbits of radius $r$ the possible values of $r$ are restricted by $L=m r^{2} \omega=$ $n(\hbar=1$ since we are employing natural units.), so that

$$
\begin{aligned}
& \omega_{n}=\frac{(G M)^{2} m^{3}}{n^{2}}, \\
& r_{n}=\frac{n^{2}}{G M m^{2}}, \\
& E_{n}=\frac{(G M)^{2} m^{3}}{2 n^{2}} .
\end{aligned}
$$

From the equations above, we see that lowest Bohr radius varies as $1 / \mathrm{m}^{2}$, and the orbital frequency as $\mathrm{m}^{3}$. As a consequence, it would be trivial to tell the mass of the orbiting particle merely by observing its radius. This result, of course, is in contradiction with what is expected from Newtonian gravity and the CEP. Nonetheless, there is no conflict between this result and the WEP. In fact, the WEP, as we have already mentioned, is a pure local statement, while Greenberger gravitational Bohr atom is an object extended in space. Note however that the gravitational Bohr atom is not a fully quantum system but only a semiquantum or semiclassical one, exactly as it happens with the original Bohr's atom model, where according to the aforementioned postulate the orbiting object has a well definite trajectory and in addition there is the extra ad hoc assumption of quantization of the angular momentum. In a fully quantum mechanical treatment, a probability of presence is obtained via the wave-function, the 'uncertainty principle' expressing the link between the width of the mentioned wave-function in both the direct and reciprocal spaces.

Note, however, that the system composed by $m$ and $M$ is very unstable. Indeed, from (1) we promptly obtain that $r \sim 1.1 \times 10^{31} \mathrm{~cm}$, where $r$ is the gravitational Bohr radius. This enormous value for $r$ is larger than the size of the observable universe and is considered as a solid proof that atoms are not governed by gravitational interactions. Nevertheless, a discrete fractal cosmological paradigm asserts that the gravitational coupling constant that applies within Atomic Scale systems is roughly 38 orders of magnitude larger than the conventional gravitational constant. According to calculations based on predictions of this discrete fractal paradigm, the value of the gravitational Bohr atom is actually on the order of $2 \pi a_{0}$, where $a_{0}$ is the standard Bohr radius. Therefore, if the prediction of the discrete fractal paradigm is taken into account, the gravitational Bohr atom is stable [36].

2.2. Tree-Level Deflection of Different Quantum Particles by an External Weak Higher-Order Gravitational Field. The action for higher-order gravity can be written as

$$
I=\int \sqrt{-g}\left[\frac{2}{\kappa^{2}} R+\frac{\alpha}{2} R^{2}+\frac{\beta}{2} R_{\mu \nu}^{2}\right]-I_{\mathrm{M}},
$$

where $\kappa^{2}=32 \pi G$, with $G$ being Newton's constant, $\alpha$ and $\beta$ are free dimensionless coefficients, and $I_{\mathrm{M}}$ is the action for matter.

The field equations concerning the action above are

$$
\begin{aligned}
& \frac{2}{\kappa^{2}} G_{\mu \nu}+\frac{\beta}{2}\left[-\frac{1}{2} g_{\mu \nu} R_{\rho \lambda}^{2}+\nabla_{\mu} \nabla_{\nu} R+2 R_{\mu \rho \lambda \nu} R^{\rho \lambda}\right. \\
& \left.-\frac{1}{2} g_{\mu \nu} \square R-\square R_{\mu \nu}\right]+\frac{\alpha}{2}\left[-\frac{1}{2} g_{\mu \nu} R^{2}+2 R R_{\mu \nu}\right. \\
& \left.\quad+2 \nabla_{\mu} \nabla_{\nu} R-2 g_{\mu \nu} \square R\right]+\frac{1}{2} \Theta_{\mu \nu}=0,
\end{aligned}
$$

where $\Theta_{\mu \nu}$ is the energy-momentum tensor.

From the above equation we promptly obtain its linear approximation doing exactly as in Einstein's theory; i.e., we write

$$
g_{\mu \nu}=\eta_{\mu \nu}+\kappa h_{\mu \nu}
$$

and then linearize the equation at hand via (4), which results in the following:

$$
\begin{aligned}
(1- & \left.\frac{\beta \kappa^{2}}{4} \square\right)\left[-\frac{1}{2} \square h_{\mu \nu}+\frac{1}{6 \kappa} R^{(\mathrm{lin})} \eta_{\mu \nu}\right] \\
& +\frac{1}{2}\left(\Gamma_{\mu, \nu}+\Gamma_{\nu, \mu}\right)=\frac{\kappa}{4}\left(T_{\mu \nu}-\frac{1}{3} T \eta_{\mu \nu}\right),
\end{aligned}
$$

where

$$
\begin{aligned}
R^{(\text {lin })} & =\frac{\kappa}{2} \square h-\kappa \gamma_{, \mu \nu}^{\mu \nu}, \\
\gamma_{\mu \nu} & \equiv h_{\mu \nu}-\frac{1}{2} \eta_{\mu \nu} h, \\
\Gamma_{\mu} & \equiv\left(1-\frac{\beta \kappa^{2}}{4} \square\right) \gamma_{\mu \nu}{ }^{, \nu}-\left(\alpha+\frac{\beta}{2}\right) \frac{\kappa}{2} R_{, \mu}^{(\operatorname{lin})} .
\end{aligned}
$$

Note that indices are raised (lowered) using $\eta^{\mu \nu}\left(\eta_{\mu \nu}\right)$. Here $T_{\mu \nu}$ is the energy-momentum tensor of special relativity. 
TABLE 1: Unpolarized differential cross sections for the tree-level scattering of different quantum particles by an external weak higher-order gravitational field, where $\theta$ is the scattering angle.

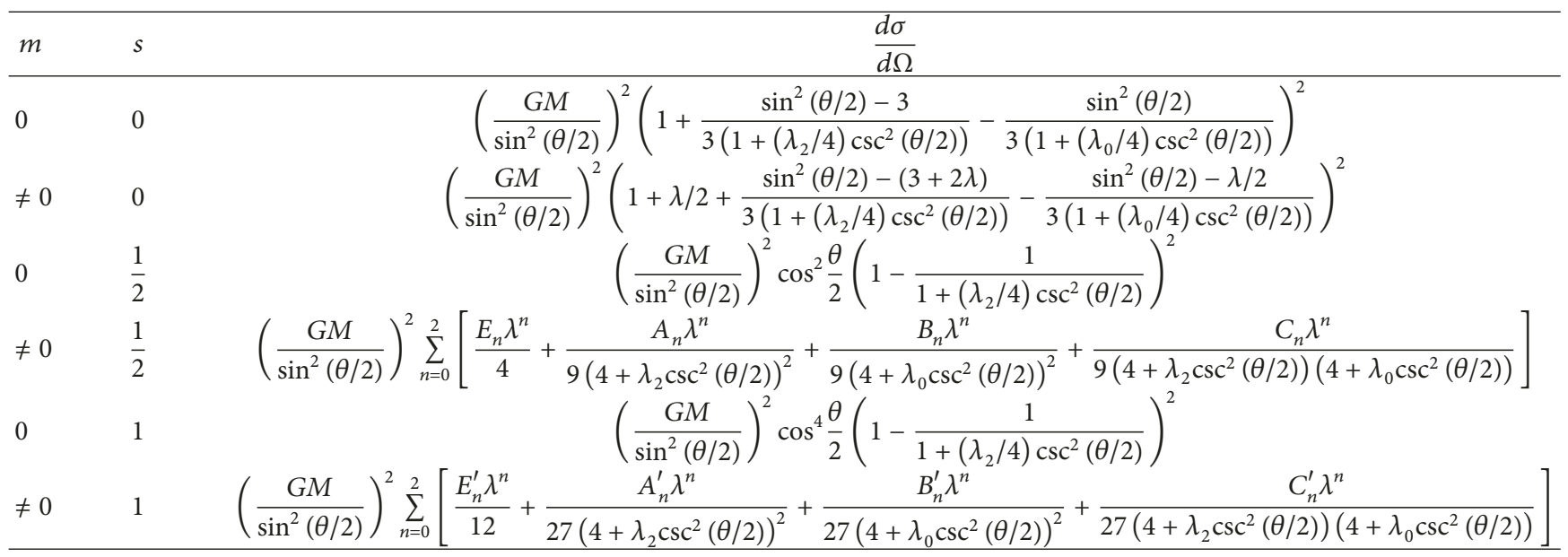

It can be shown that it is always possible to choose a coordinate system such that the gauge conditions, $\Gamma_{\mu}=0$, on the linearized metric hold. Assuming that these conditions are satisfied, it is straightforward to show that the general solution of the linearized field equations is given by the following $[37,38]$ :

$$
h_{\mu \nu}=h_{\mu \nu}^{(\mathrm{E})}-\phi \eta_{\mu \nu}+\psi_{\mu \nu}
$$

where $h_{\mu \nu}^{(\mathrm{E})}$ is the solution of linearized Einstein's equations in the de Donder gauge, i.e.,

$$
\begin{aligned}
\square h_{\mu \nu}^{(\mathrm{E})} & =\frac{\kappa}{2}\left[\frac{T \eta_{\mu \nu}}{2}-T_{\mu \nu}\right], \quad \gamma_{\mu \nu}^{(\mathrm{E}), \nu}=0, \\
\gamma_{\mu \nu}^{(\mathrm{E})} & \equiv h_{\mu \nu}^{(\mathrm{E})}-\frac{1}{2} \eta_{\mu \nu} h^{(\mathrm{E})},
\end{aligned}
$$

while $\phi$ and $\psi_{\mu \nu}$ satisfy, respectively, the following:

$$
\begin{aligned}
\left(\square+m_{0}^{2}\right) \phi & =\frac{\kappa T}{12}, \\
\left(\square+m_{2}^{2}\right) \psi_{\mu \nu} & =\frac{\kappa}{2}\left[T_{\mu \nu}-\frac{1}{3} T \eta_{\mu \nu}\right], \quad \square \psi=\psi_{\mu \nu}{ }^{, \mu \nu} .
\end{aligned}
$$

It is worthy of note that in this very special gauge the equations for $\psi_{\mu \nu}, \phi$, and $h_{\mu \nu}^{(\mathrm{E})}$ are totally decoupled. As a result, the general solution to the linearized field equations reduces to an algebraic sum of the solutions of the equations concerning the three mentioned fields.

Solving the preceding equations for a point-like particle of mass $M$ located at $\mathbf{r}=\mathbf{0}$ and having, as a consequence, an energy-momentum tensor $T_{\mu \nu}=M \eta_{\mu 0} \eta_{\nu 0} \delta^{3}(\mathbf{r})$, we find

$$
h_{\mu \nu}(r)=h_{\mu \nu}^{(\mathrm{E})}(r)+h_{\mu \nu}^{\left(\mathrm{R}^{2}\right)}(r)+h_{\mu \nu}^{\left(\mathrm{R}_{\mu \nu}^{2}\right)}(r)
$$

with

$$
\begin{aligned}
h_{\mu \nu}^{(\mathrm{E})}(r) & =\frac{M \kappa}{16 \pi}\left[\frac{\eta_{\mu \nu}}{r}-\frac{2 \eta_{\mu 0} \eta_{\nu 0}}{r}\right], \\
h_{\mu \nu}^{\left(\mathrm{R}^{2}\right)}(r) & =\frac{M \kappa}{16 \pi}\left[-\frac{1}{3} \frac{e^{-m_{0} r}}{r} \eta_{\mu \nu}\right], \\
h_{\mu \nu}^{\left(\mathrm{R}_{\mu \nu}^{2}\right)}(r) & =\frac{M \kappa}{16 \pi}\left[-\frac{2}{3} \frac{e^{-m_{2} r}}{r} \eta_{\mu \nu}+2 \frac{e^{-m_{2} r}}{r} \eta_{\mu 0} \eta_{\nu 0}\right] .
\end{aligned}
$$

Note that for $m_{0}, m_{2} \longrightarrow \infty$, the above solution reproduces the solution of linearized Einstein field equations in the de Donder gauge, as it should.

On the other hand, the momentum space gravitational field, namely, $h_{\mathrm{ext}}^{\lambda \rho}(\mathbf{k})$, is defined by

$$
h_{\mathrm{ext}}^{\lambda \rho}(\mathbf{k})=\int d^{3} \mathbf{r} e^{-i \mathbf{k} \cdot \mathbf{r}} h_{\mathrm{ext}}^{\lambda \rho}(\mathbf{r})
$$

Thence,

$$
h_{\mathrm{ext}}^{\lambda \rho}(\mathbf{k})=h_{\mathrm{ext}}^{(\mathrm{E}) \lambda \rho}(\mathbf{k})+h_{\mathrm{ext}}^{\left(\mathrm{R}_{\mu \nu}^{2}\right) \lambda \rho}(\mathbf{k})+h_{\mathrm{ext}}^{\left(\mathrm{R}^{2}\right) \lambda \rho}(\mathbf{k}),
$$

with

$$
\begin{aligned}
h_{\mathrm{ext}}^{(\mathrm{E}) \mu \nu}(\mathbf{k}) & =\frac{\kappa M}{4 \mathbf{k}^{2}} \eta^{\mu \nu}-\frac{\kappa M}{2} \frac{\eta^{\mu 0} \eta^{\nu 0}}{\mathbf{k}^{2}} \\
h_{\mathrm{ext}}^{\left(\mathrm{R}_{\alpha \beta}^{2}\right) \mu \nu}(\mathbf{k}) & =-\frac{\kappa M}{6} \frac{\eta^{\mu \nu}}{\mathbf{k}^{2}+m_{2}^{2}}+\frac{\kappa M}{2} \frac{\eta^{\mu 0} \eta^{\nu 0}}{\mathbf{k}^{2}+m_{2}^{2}} \\
h_{\mathrm{ext}}^{\left(\mathrm{R}^{2}\right) \mu \nu} & (\mathbf{k})=-\frac{\kappa M}{12} \frac{\eta^{\mu \nu}}{\mathbf{k}^{2}+m_{0}^{2}} .
\end{aligned}
$$

We are now ready to compute the tree-level scattering of different quantum particles by an external weak higher-order gravitational field. Nevertheless, since these calculations are very extensive, they were put in Appendix A.

The outcome of the experiments analyzed in Appendix A is summarized in Table 1 (We point out that the constants 
TABLE 2: Tree-level unpolarized differential cross sections for the scattering of different quantum particles by an external weak Einsteinian gravitational field.

\begin{tabular}{ccc}
\hline$m$ & $s$ & $\frac{d \sigma}{d \Omega}$ \\
\hline 0 & 0 & $\left(\frac{G M}{\sin ^{2}(\theta / 2)}\right)^{2}$ \\
$\neq 0$ & 0 & $\left(\frac{G M}{\sin ^{2}(\theta / 2)}\right)^{2}\left(1+\frac{\lambda}{2}\right)^{2}$ \\
0 & $\frac{1}{2}$ & $\left(\frac{G M}{\sin ^{2}(\theta / 2)}\right)^{2} \cos ^{2} \frac{\theta}{2}$ \\
$\neq 0$ & $\frac{1}{2}$ & $\left(\frac{G M}{\sin ^{2}(\theta / 2)}\right)^{2}\left[\cos ^{2} \frac{\theta}{2}+\frac{\lambda}{4}\left(1+\lambda+3 \cos ^{2} \frac{\theta}{2}\right)\right]$ \\
0 & 1 & $\left(\frac{G M}{\sin ^{2}(\theta / 2)}\right)^{2} \cos ^{4} \frac{\theta}{2}$ \\
$\neq 0$ & 1 & $\left(\frac{G M}{\sin ^{2}(\theta / 2)}\right)^{2}\left[\frac{1}{3}+\frac{2}{3} \cos ^{4} \frac{\theta}{2}-\frac{\lambda}{3}\left(1-\frac{3 \lambda}{4}-4 \cos ^{2} \frac{\theta}{2}\right)\right]$ \\
\hline
\end{tabular}

$\lambda, \lambda_{1}, \lambda_{2}, A_{0}, A_{1}, A_{2}, B_{0}, B_{1}, B_{2}, C_{0}, C_{1}, C_{2}, E_{0}, E_{1}, E_{2}, A_{0}^{\prime}, A_{1}^{\prime}$, $A_{2}^{\prime}, B_{0}^{\prime}, B_{1}^{\prime}, B_{2}^{\prime}, C_{0}^{\prime}, C_{1}^{\prime}, C_{2}^{\prime}, E_{0}^{\prime}, E_{1}^{\prime}, E_{2}^{\prime}$ in Table 1 are defined in Appendix A.). A cursory glance at this table is enough to convince us that the unpolarized differential cross sections and, of course, the deflection angles, depend on the spin and energy of the scattered particle.

Now, bearing in mind that any experiment carried out to test the bending of the quantum particles requires the knowledge of the gravitational deflection angle, which, of course, is an extended object, we come to the conclusion that these results can be correctly interpreted as a violation of the CEP (which is nonlocal) but not of the WEP (which is local).

An important question must be raised now: is it possible to recover the tree-level deflection angles related to general relativity from Table 1 ? The answer is affirmative. Indeed, in the $\lambda_{2}, \lambda_{0} \longrightarrow \infty$ limit, Table 1 reduces to Table 2 displayed below.

It is worthy of note that the unpolarized differential cross sections exhibited in Table 2, as well as the corresponding deflection angles, are dependent on the spin; in addition, for the massive particles, the bending depends on the energy as well.

Why the Einstein gravitational field perceives the spin? Because there is the presence of a momentum transfer $\mathbf{k}$ in the scattering responsible for probing the internal structure (spin) of the particle. Accordingly, Einstein's geometrical results are recovered in the $\mathbf{k} \longrightarrow 0$; in other words, in the nontrivial limit of small momentum transfer, which corresponds to a nontrivial small angle limit since $|\mathbf{k}|=2|\mathbf{p}| \sin (\theta /$ 2 ), the massive (massless) particles behave in the same way, regardless of the spin. In fact, if the spin is 'switched off', we find from Table 2 that, for $m=0$,

$$
\frac{d \sigma}{d \Omega} \sim \frac{16 G^{2} M^{2}}{\theta^{4}}
$$

while, for $m \neq 0$,

$$
\frac{d \sigma}{d \Omega} \sim \frac{16 G^{2} M^{2}}{\theta^{4}}\left(1+\frac{\lambda}{2}\right)^{2} .
$$

These differential cross sections can be related to a classical trajectory with impact parameter $b$ via the relations $b d b \sim-(d \sigma / d \Omega) \theta d \theta$. As a result, we conclude that, for $m=0$,

$$
\theta \sim \frac{4 G M}{b},
$$

and, for $m \neq 0$,

$$
\theta \sim \frac{4 G M}{b}\left(1+\frac{\lambda}{2}\right)
$$

The former equation gives the gravitational deflection angle for a massless particle, a result foreseen by Einstein a long time ago, whereas the latter just gives the prediction of general relativity for the bending of a massive particle by an external weak gravitational field [39]. The results of Table 2, in short, reproduce for small angles those predicted by Einstein's geometrical theory, confirming in this way the accuracy of our analytical computations. Note that since $\lambda \equiv$ $\mathrm{m}^{2} / \mathbf{p}^{2}=\left(1-\mathbf{v}^{2}\right) / \mathbf{v}^{2}$, with $\mathbf{v}$ being the velocity of the ingoing particle, (18) tells us that for $|\mathbf{v}| \ll 1, \theta \longrightarrow 2 G M / b \mathbf{v}^{2}$, which is nothing but Newton's prediction for the gravitational deflection angle; this equation reproduces also (17) in the $|\mathbf{v}| \longrightarrow 1$ limit. Interestingly enough, since $\lambda \equiv \mathrm{m}^{2} / \mathbf{p}^{2}=$ $m^{2} /\left(E^{2}-m^{2}\right)$, for $m / E \ll 1$, (18) leads to the following result:

$$
\theta \sim \frac{4 G M}{b}\left(1+\frac{m^{2}}{2 E^{2}}\right),
$$

which was recently utilized to find an upper bound on the photon mass [40-42].

\section{Violations of the CEP but Not of the WEP at the Quantum Level}

We discuss below two interesting quantum violations of the CEP but not of the WEP in the Earth gravitational field.

3.1. COW Experiment. By the mid-1970s, a few years after the publication of Greenberger's article, using a neutron interferometer, Collela, Overhauser, and Werner [7] analyzed the quantum mechanical shift of the neutrons caused by the interaction with Earth's gravitational field. Let us then compute the mentioned phase shift. To accomplish this task, we make use of a nonintegrable phase shift approach to gravitation built out utilizing the similarity of teleparallel gravity with electromagnetism [43].

Electromagnetism, as is well known, possesses in addition to the usual differential formalism also a global formulation in terms of a nonintegrable phase factor [44]. Accordingly, it can be considered as the gauge-invariant action of a nonintegrable (path-dependent) phase factor. As a result, for a particle with electric charge $e$ traveling from an initial point $\mathrm{P}$ to a final point $\mathrm{Q}$, the phase factor assumes the following form:

$$
\Phi_{e}(P \mid Q)=\exp \left[i e \int_{P}^{Q} A_{\mu} d x^{\mu}\right]
$$




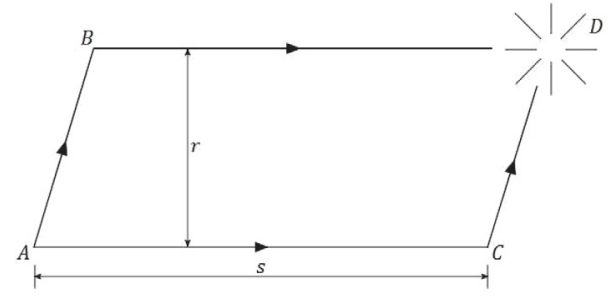

FIGURE 2: Experiment to detect gravity-induced quantum interference.

where $A_{\mu}$ is the electromagnetic gauge potential. Note that the electromagnetic phase factor can also be written as

$$
\Phi_{e}(P \mid Q)=\exp \left[i S_{e}\right]
$$

where $S_{e}$ is the action integral describing the interaction of the charged particle with the electromagnetic field.

Now, in the teleparallel approach to gravity, the fundamental field describing gravitation is the translational gauge potential $B^{a}{ }_{\mu}$. Consequently, the action integral concerning the interaction of a particle of mass $m$ with a gravitational field is given by the following [45]:

$$
S_{g}=\int_{P}^{Q} m B^{a} u_{a} d x^{\mu}
$$

So, the corresponding gravitational nonintegrable phase factor turns out to be

$$
\Phi_{g}(P \mid Q)=\exp \left[i m \int_{P}^{Q} B^{a}{ }_{\mu} u_{a} d x^{\mu}\right]
$$

It is worth mentioning that, similar to the electromagnetic phase factor, it represents the quantum mechanical law that replaces the classical gravitational Lorentz force equation [46].

Keeping in mind that a Newtonian gravitational field is characterized by the condition that only $B^{0}{ }_{0} \neq 0$, and taking into account the fact that $u^{0}=\gamma \simeq 1$ for thermal neutrons, the gravitational phase factor becomes

$$
\Phi_{g}(P \mid Q)=\exp \left[m \int_{P}^{Q} B_{00} d t\right] .
$$

In the Newtonian approximation the above expression reduces to

$$
\Phi_{g}(P \mid Q)=\exp \left[i m g \int_{P}^{Q} z(t) d t\right] \equiv \exp i \phi,
$$

where $g$ is the gravitational acceleration and $z$ is the distance from the Earth taken from some reference point.

We are now ready to calculate the phase $\phi$ through the two trajectories of Figure 2, assuming that the segment $\mathrm{AC}$ is at $z=0$. For trajectory ACD we promptly obtain

$$
\phi_{\mathrm{ACD}}=m g \int_{C}^{D} z(t) d t .
$$

Trajectory ABD gives in turn

$$
\phi_{\mathrm{ABD}}=m g \int_{A}^{B} z(t) d t+m g r \int_{B}^{D} d t .
$$

Bearing in mind that the neutron velocity is constant along the segment $\mathrm{BD}$, we find that

$$
\int_{B}^{D} d t \equiv \frac{s}{v}=\frac{s m \lambda}{2 \pi}
$$

where $\lambda=2 \pi / m v$ is the de Broglie wavelength related to the neutron.

Therefore,

$$
\Delta \phi=\phi_{\mathrm{ABD}}-\phi_{\mathrm{ACD}}=\frac{r s g \lambda m^{2}}{2 \pi} .
$$

So, we come to the conclusion that the phase shift obtained in COW experiment is dependent on the neutron mass. This landmark experiment reflects a divergence between the CEP and quantum mechanics. Note, however, that COW phase shift between the two neutron paths in which these particles are traveling at different heights in a gravitational field depends on the (macroscopic) area of the quadrilateral formed by the neutron paths, being as a consequence a nonlocal effect. Thus, COW experiment does not violate the WEP.

We call attention to the fact that more recent and more accurate experiments have been performed since COW experiment (1975) in order to test the WEP on microscopic system via atom interferometry $[47,48]$. Again, these experiments are in accordance with the WEP (they are nonlocal) but disagree with the CEP.

3.2. Free Fall in a Constant Gravitational Field of a Massive Object Described by Its Wave-Function $\Psi$. Consider now the interesting but simple case of free fall in a constant gravitational field of a massive object quantum mechanical described by its wave-function $\Psi$. We suppose that the wavefunction is initially Gaussian.

In this case the Schrödinger equation must be satisfied with the Hamiltonian

$$
H=\frac{p^{2}}{2 m}+m g z .
$$

The time of flight of the particle at hand can be computed from some initial position $z_{0}$ up to $z=0$, where the initial position is determined by the expectation value $z_{0}=$ $\left\langle\Psi_{0}|z| \Psi_{0}\right\rangle$ of the position in the Gaussian initial state $\Psi_{0}$. Now, although the time of flight is statically distributed with the mean value agreeing with the classical universal value

$$
T=\sqrt{\frac{2 z_{0}}{g}},
$$

the standard deviation of the measured values of the time of flight around $T$ depends on the mass of the particle

$$
\sigma=\frac{2 \pi}{\Delta_{0} m g},
$$

$\Delta_{0}$ being the width of the initial Gaussian wave packet. 
Therefore, we arrive at the conclusion that in this sense the quantum motion of the particle is nonuniversal since it depends on the value of its mass, which of course violates the CEP but not the WEP (the particle is an object extended in space).

\section{Final Remarks}

Two semiclassical examples that violate the CEP but not the WEP in an external gravitational field were discussed from a theoretical point of view: Greenberger gravitational Bohr atom and the deflection at the tree level of different quantum particles owed to an external weak higher-order gravitational field. In this latter case the bending is dependent on the spin and energy of the scattered particle. We analyzed also an experiment similar to the one just described where the external weak higher-order gravitational field is replaced with an external weak Einsteinian gravitational field which also violates the CEP but not the WEP.

Two quantum examples that also agree with the WEP but are not in accordance with the CEP were analyzed afterwards: COW experiment and free fall in a constant gravitational field of a massive object described by its wave-function $\Psi$.

Now, among the four examples studied in this work, only one is based on an experimental test: COW experiment. For this reason we shall elaborate a bit more on the aforementioned test. Although COW experiment was conducted in 1975, a more accurate version of it was performed in 1997 [49], and its authors reported that in this experiment the gravitationally induced phase shift of the neutron was measured with a statistical uncertainty of order 1 part in 1000 in two different interferometers. A discrepancy between the theoretically predicted and experimentally measured value of the phase shift due to gravity was also observed at the $1 \%$ level. Extensions to the theoretical description of the shape of a neutron interferogram as function of the tilt in a gravitational field were discussed and compared with experiment as well. It is worth noting that past experiments have verified the quantum mechanical equivalence of gravitational and inertial masses to a precision of about $1 \%$.

We call attention to the fact that a phase shift of the form given in (29) would be predicted for a quantum mechanical particle in the presence of any scalar potential; in our case it is the Newtonian gravitational potential. In order to fully describe this effect we need only quantum mechanics and Newton theory. Therefore, no metric description of gravity is necessary. This phenomenon, of course, is unexplainable by classical Newtonian gravity. Undoubtedly, COW experiment represents the first evidence of gravity interacting in a truly quantum mechanical way. Nevertheless, from the viewpoint of quantum theory, this effect is well understood as a scalar Aharonov-Bohm effect and manifests similarly for electric charges in electric potentials $[50,51]$.

We point out that [52-55] may be helpful for those interested in investigations similar in a sense to those dealt with in the present work.

Last but not least, we would like to draw the reader's attention to the fact that the examples discussed in this article

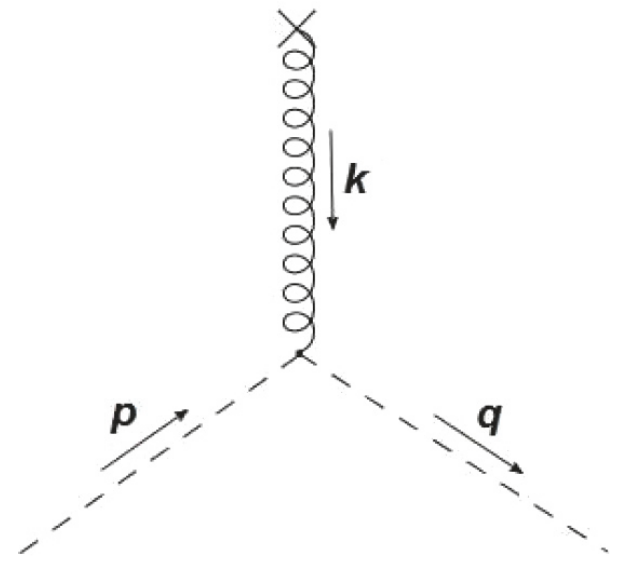

FIgURE 3: Feynman diagram for the interaction between a spin-0 particle and an external weak gravitational field.

seem to indicate that, at first sight, the only possibility of violating the WEP is through local experiments.

The authors are very grateful to FAPERJ and $\mathrm{CNPq}$ for their financial support.

\section{Appendix}

\section{A. Unpolarized Differential Cross Sections for Tree-Level Scattering of Different Quantum Particles by an External Weak Higher-Order Gravitational Field}

A.1. Spin-0 Particles. The Lagrangian for a massive scalar field minimally coupled to gravity can be written as

$$
\mathscr{L}=\frac{\sqrt{-g}}{2}\left(g^{\mu \nu} \partial_{\mu} \phi \partial_{\nu} \phi-m^{2} \phi^{2}\right),
$$

and leads to first order in $k$ to the following Lagrangian for the interaction of a scalar field with a weak gravitational field:

$$
\mathscr{L}_{\text {int }}=-\frac{\kappa}{2} h^{\mu \nu}\left[\partial_{\mu} \phi \partial_{\nu} \phi-\frac{1}{2} \eta_{\mu \nu}\left(\partial_{\alpha} \phi \partial^{\alpha} \phi-m^{2} \phi^{2}\right)\right] .
$$

From the preceding Lagrangian we promptly obtain the vertex for the process depicted in Figure 3:

$$
\begin{aligned}
& V(\mathbf{p}, \mathbf{q}) \\
& \quad=-\frac{\kappa}{2} h_{\mathrm{ext}}^{\mu \nu}(\mathbf{k})\left[p_{\mu} q_{\nu}+p_{\nu} q_{\mu}-\eta_{\mu \nu}\left(p \cdot q-m^{2}\right)\right],
\end{aligned}
$$

where the external field is a weak higher-order gravitational field.

Now, the differential cross section for the process above reads

$$
\frac{d \sigma}{d \Omega}=\frac{|\mathscr{M}|^{2}}{(4 \pi)^{2}}
$$

where the Feynman amplitude $\mathscr{M}$ coincides with $V(\mathbf{p}, \mathbf{q})$. 
Accordingly, the differential cross section for the treelevel scattering of a massive spin-0 particle by an external weak higher-derivative gravitational field assumes the following form:

$$
\begin{gathered}
\frac{d \sigma}{d \Omega}=\left[\frac{G M}{\sin ^{2}(\theta / 2)}\right]^{2}\left[1+\frac{\lambda}{2}\right. \\
+\frac{\sin ^{2}(\theta / 2)-(3+2 \lambda)}{3\left(1+(1 / 4) \lambda_{2} \csc ^{2}(\theta / 2)\right)} \\
\left.+\frac{(1 / 2) \lambda-\sin ^{2}(\theta / 2)}{3\left(1+(1 / 4) \lambda_{0} \csc ^{2}(\theta / 2)\right)}\right]^{2},
\end{gathered}
$$

where

$$
\begin{gathered}
\lambda \equiv \frac{m^{2}}{\mathbf{p}^{2}}, \\
\lambda_{2} \equiv \frac{M_{2}^{2}}{\mathbf{p}^{2}}, \\
\lambda_{0} \equiv \frac{M_{0}^{2}}{\mathbf{p}^{2}} .
\end{gathered}
$$

Now, since $E^{2}=\mathbf{p}^{2}(1+\lambda)$ where $E$ is the particle energy, $\lambda_{2}$ and $\lambda_{0}$ can be written as

$$
\begin{aligned}
& \lambda_{2}=\frac{(1+\lambda) M_{2}^{2}}{E^{2}}, \\
& \lambda_{0}=\frac{(1+\lambda) M_{0}^{2}}{E^{2}},
\end{aligned}
$$

which clearly shows that all the parameters in (A.6) are energy-dependent.

In the $m \longrightarrow 0$ limit, we get the differential cross section for tree-level scattering of a massless spin-0 boson by an external weak higher-derivative gravitational field:

$$
\begin{aligned}
\frac{d \sigma}{d \Omega} & =\left[\frac{G M}{\sin ^{2}(\theta / 2)}\right]^{2}[1 \\
& +\frac{\sin ^{2}(\theta / 2)-3}{3\left(1+(1 / 4) \lambda_{2} \csc ^{2}(\theta / 2)\right)} \\
& \left.-\frac{\sin ^{2}(\theta / 2)}{3\left(1+(1 / 4) \lambda_{0} \csc ^{2}(\theta / 2)\right)}\right]^{2} .
\end{aligned}
$$

Note that if $m=0, \lambda_{2}=M_{2}^{2} / E^{2}$ and $\lambda_{0}=M_{0}^{2} / E^{2}$. Here we are using the same symbols for denoting the parameters $\lambda_{2}$ and $\lambda_{0}$ as those utilized for the massive case since their meaning are quite clear from the context. Therefore, from now on these symbols will utilized for both massive and massless particles.

A.2. Spin 1/2 Particles. As is well known, the gravitational Lagrangian for a massive fermion is given by the following [56]:

$$
\mathscr{L}=\sqrt{-g}\left[\frac{i}{2}\left(\bar{\psi} \gamma^{\mu} \vec{\nabla}_{\mu} \psi-\bar{\psi} \overleftarrow{\nabla_{\mu}} \gamma^{\mu} \psi\right)-m \bar{\psi} \psi\right]
$$

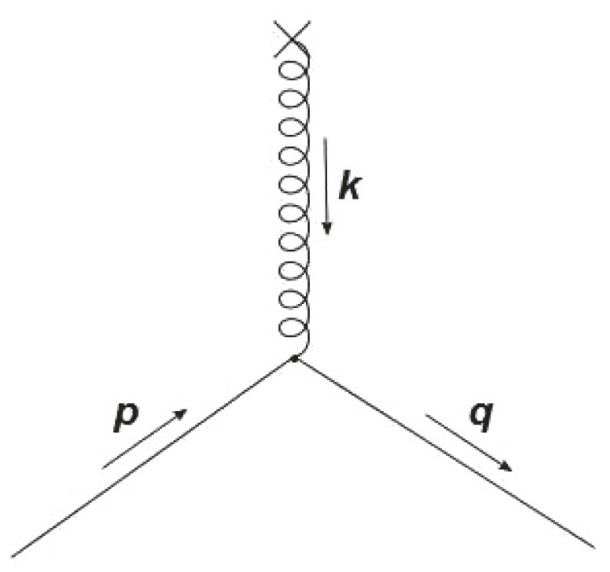

FIGURE 4: Diagram for the interaction of a fermion with an external weak gravitational field.

with the notation

$$
\begin{gathered}
\gamma^{\mu}=\gamma^{p} e_{p}^{\mu}, \\
\vec{\nabla}_{\mu} \psi=\partial_{\mu} \psi+i w_{\mu} \psi, \\
\bar{\psi} \overleftarrow{\nabla}_{\mu}=\partial_{\mu} \bar{\psi}-i \bar{\psi} w_{\mu} .
\end{gathered}
$$

Here $e_{n}^{v} \equiv \eta_{m n} g^{\mu v} e_{\mu}^{m}(x)$ is a different type of vierbein where the $m$ index is lowered with the Minkowski metric $\eta_{n m}$, while the $\mu$ index is raised with $g^{\mu \nu}$, whereas the field connection $w_{\mu}(x)$ is expressed in terms of the tetrads as

$$
\begin{aligned}
& w_{\mu}(x)=\frac{1}{4} \sigma^{m n}\left[e_{m}^{\nu}\left(\partial_{\mu} e_{n \nu}-\partial_{\nu} e_{n \mu}\right)\right. \\
& \left.\quad+\frac{1}{2} e_{m}^{\rho} e_{n}^{\sigma}\left(\partial_{\sigma} e_{l \rho}-\partial_{\rho} e_{l \sigma}\right) e_{\mu}^{l}-(m \longleftrightarrow n)\right],
\end{aligned}
$$

where the Dirac matrices are denoted by $\gamma^{n}$, and $\sigma^{m n}=(i /$ 2) $\left[\gamma^{m}, \gamma^{n}\right]$.

Keeping in mind that to order $k[57,58]$,

$$
e_{\mu}^{m}=\delta_{\mu}^{m}+\frac{\kappa}{2} h_{\mu}^{m}+\mathcal{O}\left(k^{2}\right),
$$

we find that within this approximation the Lagrangian for the interaction of a fermion with a weak gravitational field has the following form:

$$
\begin{aligned}
& \mathscr{L}_{\text {int }}=-\frac{\kappa}{2} h_{\mu \nu}\left\{\frac { i } { 2 } \left[\left(\bar{\psi} \gamma^{\mu} \partial^{\nu} \psi-\partial^{\nu} \bar{\psi} \gamma^{\mu} \psi\right)\right.\right. \\
& \left.\left.-\eta^{\mu \nu}\left(\bar{\psi} \gamma^{\alpha} \partial_{\alpha} \psi-\partial_{\alpha} \bar{\psi} \gamma^{\alpha} \psi\right)\right]+\eta^{\mu \nu} m \bar{\psi} \psi\right\} .
\end{aligned}
$$

It follows that the vertex for the process shown in Figure 4 reads

$$
\begin{gathered}
V(\mathbf{p}, \mathbf{q})=\frac{\kappa}{8} h_{\mathrm{ext}}^{\mu \nu}(\mathbf{k})\left[2 \eta_{\mu \nu}(\not p+q-2 m)\right. \\
\left.-\gamma_{\mu}(p+q)_{\nu}-(p+q)_{\mu} \gamma_{\nu}\right],
\end{gathered}
$$

where $h_{\text {ext }}^{\mu \nu}(\mathbf{k})$ is given by (13). 
The unpolarized differential cross section for the process at hand, in turn, is given by

$$
\frac{d \sigma}{d \Omega}=\frac{(2 m)^{2}}{(4 \pi)^{2}} \frac{1}{2} \sum_{r^{\prime}, r}\left|\mathscr{M}_{r^{\prime}, r}\right|^{2},
$$

where

$$
\mathscr{M}_{r^{\prime}, r}=\bar{u}_{r^{\prime}}(\mathbf{q}) V(\mathbf{p}, \mathbf{q}) u_{r}(\mathbf{p}) .
$$

Taking the relation

$$
\sum_{r=1}^{2} u_{r}(\mathbf{p}) \bar{u}_{r}(\mathbf{p})=\frac{\not p+m}{2 m}
$$

into account, we find that the unpolarized differential cross section for the scattering of a massive fermion by an external weak higher-order gravitational field reads

$$
\begin{aligned}
\frac{d \sigma}{d \Omega} & =\left(\frac{G M}{\sin ^{2}(\theta / 2)}\right)^{2} \sum_{n=0}^{2}\left[\frac{E_{n}(\lambda)^{n}}{4}\right. \\
& +\frac{A_{n}(\lambda)^{n}}{9\left(4+\lambda_{2} \csc ^{2}(\theta / 2)\right)^{2}}+\frac{B_{n}(\lambda)^{n}}{9\left(4+\lambda_{0} \csc ^{2}(\theta / 2)\right)^{2}} \\
& \left.+\frac{C_{n}(\lambda)^{n}}{9\left(4+\lambda_{2} \csc ^{2}(\theta / 2)\right)\left(4+\lambda_{0} \csc ^{2}(\theta / 2)\right)}\right],
\end{aligned}
$$

where

$$
\begin{aligned}
E_{0}= & 4 \cos ^{2} \frac{\theta}{2}, \\
E_{1}= & 3 \cos ^{2} \frac{\theta}{2}+1, \\
E_{2}= & 1 ; \\
A_{0}= & -\left(72 \cos ^{2} \frac{\theta}{2}\right) \lambda_{2} \csc ^{2} \frac{\theta}{2}-144 \cos ^{2} \frac{\theta}{2}, \\
A_{1}= & -\left(60 \cos ^{2} \frac{\theta}{2}\right) \lambda_{2} \csc ^{2} \frac{\theta}{2}-24 \lambda_{2} \csc ^{2} \frac{\theta}{2} \\
& -112 \cos ^{2} \frac{\theta}{2}-32, \\
A_{2}= & -24 \lambda_{2} \csc ^{2} \frac{\theta}{2}-32 ; \\
B_{0}= & 0, \\
B_{1}= & \left(6 \cos ^{2} \frac{\theta}{2}\right) \lambda_{0} \csc ^{2} \frac{\theta}{2}+6 \lambda_{0} \csc ^{2} \frac{\theta}{2}+20 \cos ^{2} \frac{\theta}{2} \\
& +28, \\
C_{0}= & 0, \\
B_{2}= & 6 \lambda_{0} \csc ^{2} \frac{\theta}{2}+28 ; \\
&
\end{aligned}
$$

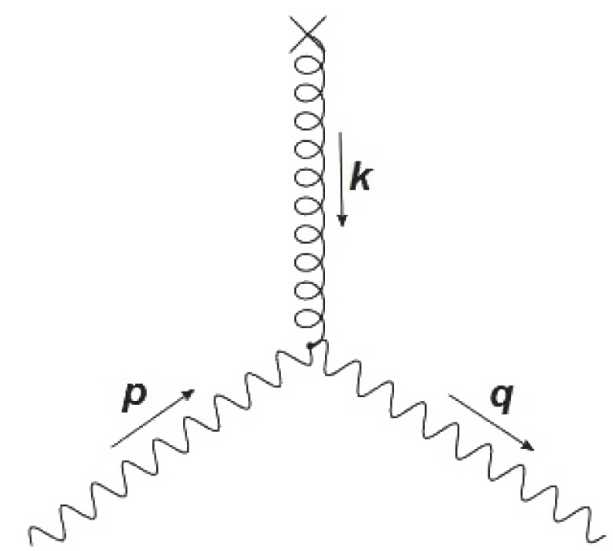

FIGURE 5: Diagram for the interaction between a photon and an external weak gravitational field.

$$
\begin{aligned}
& C_{1}=-16 \cos ^{2} \frac{\theta}{2}-32, \\
& C_{2}=-32 .
\end{aligned}
$$

In the $m \longrightarrow 0$ limit, we obtain the differential cross section for a massless fermion

$$
\begin{aligned}
\frac{d \sigma}{d \Omega}= & \left(\frac{G M}{\sin ^{2}(\theta / 2)}\right)^{2} \cos ^{2} \frac{\theta}{2} \\
& \cdot\left[1-\frac{1}{1+(1 / 4) \lambda_{2} \csc ^{2}(\theta / 2)}\right]^{2} .
\end{aligned}
$$

A.3. Spin-1 Particles. The gravitational Lagrangian for a massive photon can be written as

$$
\mathscr{L}=\sqrt{-g}\left[-\frac{1}{4} g^{\mu \alpha} g^{\nu \beta} F_{\mu \nu} F_{\alpha \beta}+\frac{m^{2}}{2} g^{\mu \nu} A_{\mu} A_{\nu}\right],
$$

from which we trivially obtain the Lagrangian for the interaction of a massive photon with a weak gravitational field:

$$
\begin{gathered}
\mathscr{L}_{\text {int }}=-\frac{\kappa}{2} h^{\mu \nu}\left[\frac{1}{4} \eta_{\mu \nu} F_{\alpha \beta}^{2}-F_{\mu}^{\alpha} F_{\nu \alpha}\right. \\
\left.+m^{2}\left(A_{\mu} A_{\nu}-\frac{1}{2} \eta_{\mu \nu} A_{\alpha}^{2}\right)\right] .
\end{gathered}
$$

Accordingly, the vertex for process represented in Figure 5 is given by

$$
\begin{aligned}
V_{\alpha \beta}(\mathbf{p}, \mathbf{q})=-\frac{\kappa}{2} h_{\mathrm{ext}}^{\mu \nu}(\mathbf{k}) \\
\cdot\left[\left(\eta_{\alpha \beta} \eta_{\mu \nu}-\eta_{\alpha \mu} \eta_{\beta \nu}-\eta_{\alpha \nu} \eta_{\beta \mu}\right)\left(p \cdot q-m^{2}\right)\right. \\
-\eta_{\alpha \beta} p_{\nu} q_{\mu}+\eta_{\mu \beta} p_{\nu} q_{\alpha}-\eta_{\mu \nu} p_{\beta} q_{\alpha}+\eta_{\alpha \nu} p_{\beta} q_{\mu} \\
\left.+\eta_{\beta \nu} p_{\alpha} q_{\mu}-\eta_{\mu \nu} p_{\alpha} q_{\beta}+\eta_{\alpha \mu} p_{\nu} q_{\beta}\right],
\end{aligned}
$$

where the external field is a weak higher-order gravitational field. 
Therefore, the unpolarized differential cross section for the process above can be written as

$$
\frac{d \sigma}{d \Omega}=\frac{1}{(4 \pi)^{2}} \frac{1}{3} \sum_{r^{\prime}, r}\left|\mathscr{M}_{r^{\prime}, r}\right|^{2},
$$

where

$$
\mathscr{M}_{r^{\prime}, r}=\epsilon_{r^{\prime}}^{\alpha}(\mathbf{q}) V_{\alpha \beta}(\mathbf{p}, \mathbf{q}) \epsilon_{r}^{\beta}(\mathbf{p})
$$

Here $\epsilon_{r}^{\beta}(\mathbf{p})$ and $\epsilon_{r^{\prime}}^{\alpha}(\mathbf{q})$ are respectively the ingoing and outgoing photon polarizations.

Now, bearing in mind that

$$
\sum_{r=1}^{3} \epsilon_{r}^{\mu}(\mathbf{p}) \epsilon_{r}^{\nu}(\mathbf{p})=-\eta^{\mu \nu}+\frac{p^{\mu} p^{\nu}}{m^{2}}
$$

we come to the conclusion that

$$
\begin{aligned}
\frac{d \sigma}{d \Omega} & =\left(\frac{G M}{\sin ^{2}(\theta / 2)}\right)^{2} \sum_{n=0}^{2}\left[\frac{E_{n}^{\prime}(\lambda)^{n}}{12}\right. \\
& +\frac{A_{n}^{\prime}(\lambda)^{n}}{27\left(4+\lambda_{2} \csc ^{2}(\theta / 2)\right)^{2}} \\
& +\frac{B_{n}^{\prime}(\lambda)^{n}}{27\left(4+\lambda_{0} \csc ^{2}(\theta / 2)\right)^{2}} \\
& \left.+\frac{C_{n}^{\prime}(\lambda)^{n}}{27\left(4+\lambda_{2} \csc ^{2}(\theta / 2)\right)\left(4+\lambda_{0} \csc ^{2}(\theta / 2)\right)}\right]
\end{aligned}
$$

where

$$
\begin{aligned}
E_{0}^{\prime}= & 4+8 \cos ^{4} \frac{\theta}{2}, \\
E_{1}^{\prime}= & -4+16 \cos ^{2} \frac{\theta}{2} \\
E_{2}^{\prime}= & 3 ; \\
A_{0}^{\prime}= & -128-32 \cos ^{2} \frac{\theta}{2}-272 \cos ^{4} \frac{\theta}{2}+168 \lambda_{2} \\
& +144 \lambda_{2} \cos ^{2} \frac{\theta}{2}-216 \lambda_{2} \csc ^{2} \frac{\theta}{2}, \\
A_{1}^{\prime}= & 160-592 \cos ^{2} \frac{\theta}{2}+324 \lambda_{2}-252 \lambda_{2} \csc ^{2} \frac{\theta}{2}, \\
A_{2}^{\prime}= & -96-72 \lambda_{2} \csc ^{2} \frac{\theta}{2} ; \\
B_{0}^{\prime}= & -80+64 \cos ^{2} \frac{\theta}{2}+16 \cos ^{4} \frac{\theta}{2}-24 \lambda_{0}, \\
B_{1}^{\prime}= & 16+128 \cos ^{2} \frac{\theta}{2}-36 \lambda_{0}+36 \lambda_{0} \csc ^{2} \frac{\theta}{2},
\end{aligned}
$$

$$
\begin{aligned}
& B_{2}^{\prime}=84+18 \lambda_{0} \csc ^{2} \frac{\theta}{2} ; \\
& C_{0}^{\prime}=64-32 \cos ^{2} \frac{\theta}{2}-32 \cos ^{4} \frac{\theta}{2}, \\
& C_{1}^{\prime}=-32-112 \cos ^{2} \frac{\theta}{2},
\end{aligned}
$$$$
C_{2}^{\prime}=-96
$$

On the other hand, the gravitational Lagrangian for a massless photon has the following form:

$$
\mathscr{L}=\sqrt{-g}\left(\frac{1}{4} g^{\mu \alpha} g^{\nu \beta} F_{\mu \nu} F_{\alpha \beta}\right),
$$

and from it we find the Lagrangian for the interaction between a massless photon and a weak gravitational:

$$
\mathscr{L}_{\text {int }}=-\frac{\kappa}{2} h^{\mu \nu}\left(\frac{1}{4} \eta_{\mu \nu} F_{\alpha \beta}^{2}-F_{\mu}{ }^{\alpha} F_{\nu \alpha}\right) .
$$

It follows then that the vertex for the interaction of a massless photon with an external weak higher-order gravitational field reads

$$
\begin{aligned}
& V_{\alpha \beta}(\mathbf{p}, \mathbf{q})=-\frac{\kappa}{2} h_{\mathrm{ext}}^{\mu \nu}(\mathbf{k}) \\
& \cdot\left[\left(\eta_{\alpha \beta} \eta_{\mu \nu}-\eta_{\alpha \mu} \eta_{\beta \nu}-\eta_{\alpha \nu} \eta_{\beta \mu}\right) p \cdot q-\eta_{\alpha \beta} p_{\nu} q_{\mu}\right. \\
& +\eta_{\mu \beta} p_{\nu} q_{\alpha}-\eta_{\mu \nu} p_{\beta} q_{\alpha}+\eta_{\alpha \nu} p_{\beta} q_{\mu}+\eta_{\beta \nu} p_{\alpha} q_{\mu} \\
& \left.\quad-\eta_{\mu \nu} p_{\alpha} q_{\beta}+\eta_{\alpha \mu} p_{\nu} q_{\beta}\right]
\end{aligned}
$$

Now, the differential cross section for the process under discussion can be written as

$$
\frac{d \sigma}{d \Omega}=\frac{1}{(4 \pi)^{2}} \frac{1}{2} \sum_{r^{\prime}, r}\left|\mathscr{M}_{r^{\prime}, r}\right|^{2},
$$

where

$$
\mathscr{M}_{r^{\prime}, r}=\epsilon_{r^{\prime}}^{\alpha}(\mathbf{q}) V_{\alpha \beta}(\mathbf{p}, \mathbf{q}) \epsilon_{r}^{\beta}(\mathbf{p}) .
$$

Keeping in mind that

$$
\begin{aligned}
\sum_{r=1}^{2} \epsilon_{r}^{\mu}(\mathbf{p}) \epsilon_{r}^{\nu}(\mathbf{p}) & \\
= & -\eta_{\mu \nu} \\
& \quad-\frac{1}{(p \cdot n)^{2}}\left[p^{\mu} p^{\nu}-p \cdot n\left(p^{\mu} n^{\nu}+p^{\nu} n^{\mu}\right)\right],
\end{aligned}
$$

where $n^{2}=1$, we arrive at the conclusion that

$$
\begin{aligned}
\frac{d \sigma}{d \Omega}= & \left(\frac{G M}{\sin ^{2}(\theta / 2)}\right)^{2} \cos ^{4} \frac{\theta}{2} \\
& \cdot\left(1-\frac{1}{1+(1 / 4) \lambda_{2} \sin ^{2}(\theta / 2)}\right)^{2} .
\end{aligned}
$$




\section{Conflicts of Interest}

The authors declare that there are no conflicts of interest regarding the publication of this paper.

\section{References}

[1] R. Hanson, H. Bernien, A. E. Dréau et al., "Loophole-free Bell inequality violation using electron spins separated by 1.3 kilometres," Nature, vol. 526, pp. 682-686, 2015.

[2] H. C. Ohanian and R. Ruffini, Gravitation and Spacetime, Cambridge University Press, 3rd edition, 2013.

[3] C. M. Will, "The confrontation between general relativity and experiment," Living Reviews in Relativity, vol. 17, article 4, 2014.

[4] A. Accioly and R. Paszko, "Conflict between the classical equivalence principle and quantum mechanics," Advanced Studies in Theoretical Physics, vol. 3, pp. 65-78, 2009.

[5] A. Accioly and R. Paszko, "Quantum mechanics versus equivalence principle," Physical Review D: Particles, Fields, Gravitation and Cosmology, vol. 78, no. 6, Article ID 064002, 6 pages, 2008.

[6] D. Greenberger, "The role of equivalence in Quantum Mechanics," Annals of Physics, vol. 47, pp. 116-126, 1968.

[7] R. Colella, A. W. Overhauser, and S. A. Werner, "Observation of gravitationally induced quantum interference," Physical Review Letters, vol. 34, no. 23, pp. 1472-1474, 1975.

[8] R. Aldrovandi, J. Pereira, and K. Vu, "Gravity and the quantum: are they reconcilable?” AIP Conference Proceedings, vol. 810, p. 217, 2006.

[9] R. Aldrovandi, J. Pereira, and K. Vu, "Bringing together gravity and the quanta," AIP Conference Proceedings, vol. 861, p. 277, 2006.

[10] N. Kajuri, "Strong equivalence principle in polymer quantum mechanics and deformed Heisenberg algebra," Physical Review D: Particles, Fields, Gravitation and Cosmology, vol. 94, no. 8, Article ID 084007, 8 pages, 2016.

[11] L. M. Krauss and S. Tremaine, "Test of the weak equivalence principle for neutrinos and photons," Physical Review Letters, vol. 60, no. 3, pp. 176-177, 1988.

[12] M. J. Longo, "New precision tests of the einstein equivalence principle from Sn1987a," Physical Review Letters, vol. 60, no. 3, pp. 173-175, 1988.

[13] J.-J. Wei, H. Gao, X.-F. Wu, and P. Mészáros, “Testing Einstein’s equivalence principle with fast radio bursts," Physical Review Letters, vol. 115, Article ID 261101, 5 pages, 2015.

[14] H. Yu, S. Xi, and F. Wang, "A new method to test the Einstein's Weak Equivalence Principle," https://arxiv.org/abs/1708.02396.

[15] H. Yu and F. Wang, "Testing weak equivalence principle with strongly lensed cosmic transients," https://arxiv.org/abs/ 1801.01257.

[16] K. S. Thorne, D. L. Lee, and A. P. Lightman, "Foundations for a theory of gravitation theories," Physical Review D: Particles, Fields, Gravitation and Cosmology, vol. 7, no. 12, pp. 3563-3578, 1973.

[17] H. C. Ohanian, "Comment of the Schiff conjecture," Physical Review D: Particles, Fields, Gravitation and Cosmology, vol. 10, pp. 2041-2042, 1974.

[18] W. T. Ni, "Equivalence principles and electromagnetism," Physical Review Letters, vol. 38, no. 7, pp. 301-304, 1977.

[19] A. Coley, "Schiff's conjecture on gravitation," Physical Review Letters, vol. 49, no. 12, pp. 853-855, 1982.
[20] A. Accioly, U. Wichoski, and N. Bertarello, "Counterexamples to Schiff's conjecture," Brazilian Journal of Physics, vol. 23, p. 392, 1993.

[21] M. P. Haugan, "Energy conservation and the principle of equivalence," Annals of Physics, vol. 118, no. 1, pp. 156-186, 1979.

[22] A. P. Lightman and D. L. Lee, "Restricted proof that the weak equivalence principle implies the Einstein equivalence principle," Physical Review D: Particles, Fields, Gravitation and Cosmology, vol. 8, no. 2, pp. 364-376, 1973.

[23] E. Fischbach, D. Sudarsky, A. Szafer, C. Talmadge, and S. H. Aronson, "Reanalysis of the Eoumltvös experiment," Physical Review Letters, vol. 56, no. 1, pp. 3-6, 1986.

[24] E. Fischbach, G. T. Gillies, D. E. Krause, J. G. Schwan, and C. Talmadge, "Non-newtonian gravity and new weak forces: an index of measurements and theory," Metrologia, vol. 29, pp. 213260, 1992.

[25] E. Fischbach and C. Talmadge, "Six years of the fifth force," Nature, vol. 356, no. 6366, pp. 207-215, 1992.

[26] E. Fischbach and C. L. Talmadge, The Search for Non-Newtonian Gravity, Springer, New York, NY, USA, 1999.

[27] J. C. Long, H. W. Chan, and J. C. Price, "Experimental status of gravitational-strength forces in the sub-centimeter regime," Nuclear Physics B, vol. 539, no. 1-2, pp. 23-34, 1999.

[28] C. Hoyle, U. Schmidt, B. R. Heckel et al., "Submillimeter test of the gravitational inverse-square law: a search for "large" extra dimensions," Physical Review Letters, vol. 86, no. 8, pp. 14181421, 2001.

[29] C. D. Hoyle, D. J. Kapner, B. R. Heckel et al., "Submillimeter tests of the gravitational inverse-square law," Physical Review D, vol. 70, Article ID 042004, 2004.

[30] K. S. Stelle, "Renormalization of higher-derivative quantum gravity," Physical Review D: Particles, Fields, Gravitation and Cosmology, vol. 16, no. 4, pp. 953-969, 1977.

[31] A. Accioly, G. Correia, G. P. de Brito, J. de Almeida, and W. Herdy, "Relating renormalizability of $D$-dimensional higherorder electromagnetic and gravitational models to the classical potential at the origin," Modern Physics Letters A, vol. 32, no. 8, Article ID 1750048, 26 pages, 2017.

[32] A. Accioly, J. de Almeida, G. P. Brito, and G. Correia, "Renormalizability in D-dimensional higher-order gravity," Physical Review D: Particles, Fields, Gravitation and Cosmology, vol. 95, Article ID 084007, 2017.

[33] I. L. Shapiro, A. M. Pelinson, and F. Salles, "Gravitational waves and perspectives for quantum gravity," Modern Physics Letters A, vol. 29, no. 30, Article ID 1430034, 21 pages, 2014.

[34] T. P. Sotiriou and V. Faraoni, " $f(R)$ theories of gravity," Reviews of Modern Physics, vol. 82, no. 1, pp. 451-497, 2010.

[35] A. Accioly, J. Helayël-Neto, B. Giacchini, and W. Herdy, "Classical and tree-level approaches to gravitational deflection in higher-derivative gravity," Physical Review D: Particles, Fields, Gravitation and Cosmology, vol. 91, no. 12, Article ID 125009, 9 pages, 2015.

[36] R. Oldershaw, “The gravitational bohr radius,” https://arxiv.org/ abs/0803.1197.

[37] P. Teyssandier, "Linearised $R+R^{2}$ gravity: a new gauge and new solutions," Classical and Quantum Gravity, vol. 6, no. 2, pp. 219229, 1989.

[38] A. Accioly, A. Azeredo, H. Mukai, and E. de Rey Neto, "Scattering of photons by an external gravitational field in the framework of higher-derivative gravity," Progress of Theoretical Physics, vol. 104, no. 1, pp. 103-122, 2000. 
[39] A. Accioly and S. Ragusa, "Gravitational deflection of massive particles in classical and semiclassical general relativity," Classical and Quantum Gravity, vol. 19, no. 21, pp. 5429-5434, 2002, Corrigendum: Classical and Quantum Gravity, vol. 20, p. 4963, 2003.

[40] A. Accioly, J. Helayël-Neto, and E. Scatena, "Combining general relativity, massive QED and Very Long Baseline Interferometry to gravitationally constrain the photon mass," Physics Letters A, vol. 374, no. 37, pp. 3806-3809, 2010.

[41] A. Accioly, J. Helayël-Neto, R. Turcati, J. Morais, and E. Scatena, "Gravitational and quantum bounds on the photon mass," Classical and Quantum Gravity, vol. 27, no. 20, Article ID 205010, 8 pages, 2010.

[42] A. Accioly, J. Helayël-Neto, and E. Scatena, "Upper bounds on the photon mass," Physical Review D: Particles, Fields, Gravitation and Cosmology, vol. 82, no. 6, Article ID 065026, 2010.

[43] R. Aldrovandi, J. G. Pereira, and K. H. Vu, "Gravitation: global formulation and quantum effects," Classical and Quantum Gravity, vol. 21, no. 1, pp. 51-62, 2004.

[44] T. T. Wu and C. N. Yang, "Concept of nonintegrable phase factors and global formulation of gauge fields," Physical Review D: Particles, Fields, Gravitation and Cosmology, vol. 12, no. 12, pp. 3845-3857, 1975.

[45] R. Aldrovandi, J. G. Pereira, and K. H. Vu, "Gravitation without the equivalence principle," General Relativity and Gravitation, vol. 36, no. 1, pp. 101-110, 2004.

[46] R. Aldrovandi and J. G. Pereira, Teleparallel Gravity, vol. 173 of Fundamental Theories of Physics, Springer, 2013.

[47] A. Peters, K. Chung, and S. Chu, "Measurement of gravitational acceleration by dropping atoms," Nature, vol. 400, pp. 849-852, 1999.

[48] S. Merlet, Q. Bodart, N. Malossi et al., "Comparison between two mobile absolute gravimeters: optical versus atomic interferometers," Metrologia, vol. 47, no. 4, pp. L9-L11, 2010.

[49] K. C. Littrell, B. E. Allman, and S. A. Werner, "Two-wavelengthdifference measurement of gravitationally induced quantum interference phases," Physical Review A: Atomic, Molecular and Optical Physics, vol. 56, no. 3, pp. 1767-1780, 1997.

[50] M. Zych, F. Costa, I. Pikovski, and C. Brukner, "Quantum interferometric visibility as a witness of general relativistic proper time," Nature Communications, vol. 2, article 505, 2011.

[51] B. E. Allman, W. Lee, O. I. Motrunich, and S. A. Werner, "Scalar Aharonov-Bohm effect with longitudinally polarized neutrons," Physical Review A: Atomic, Molecular and Optical Physics, vol. 60, no. 6, pp. 4272-4284, 1999.

[52] G. Rosi, G. D’Amico, L. Cacciapuoti et al., "Quantum test of the equivalence principle for atoms in coherent superposition of internal energy states," Nature Communications, vol. 8, Article ID 15529, 2017.

[53] M. Zich and C. Brukner, "Quantum formulation of the Einstein equivalence principle," https://arxiv.org/abs/1502.00971v1.

[54] P. Orlando, R. Mann, K. Modi, and F. Pollock, "A simple test of the equivalence principle(s) for quantum superpositions," Classical and Quantum Gravity, vol. 33, Article ID 19LT01, 2016.

[55] P. J. Orlando, F. A. Pollock, and K. Modi, "How does interference fall?" in Lectures on General Quantum Correlations and their Applications. Quantum Science and Technology, pp. 421-451, Springer, 2017.

[56] S. Y. Choi, J. S. Shim, and H. S. Song, "Factorization and polarization in linearized gravity," Physical Review D: Particles,
Fields, Gravitation and Cosmology, vol. 51, no. 6, pp. 2751-2769, 1995.

[57] R. P. Woodard, "The vierbein is irrelevant in perturbation theory," Physics Letters B, vol. 148, no. 6, pp. 440-444, 1984.

[58] H. T. Cho and K. L. Ng, "Spinor technique in graviton scattering processes," Physical Review D: Particles, Fields, Gravitation and Cosmology, vol. 47, no. 4, pp. 1692-1698, 1993. 

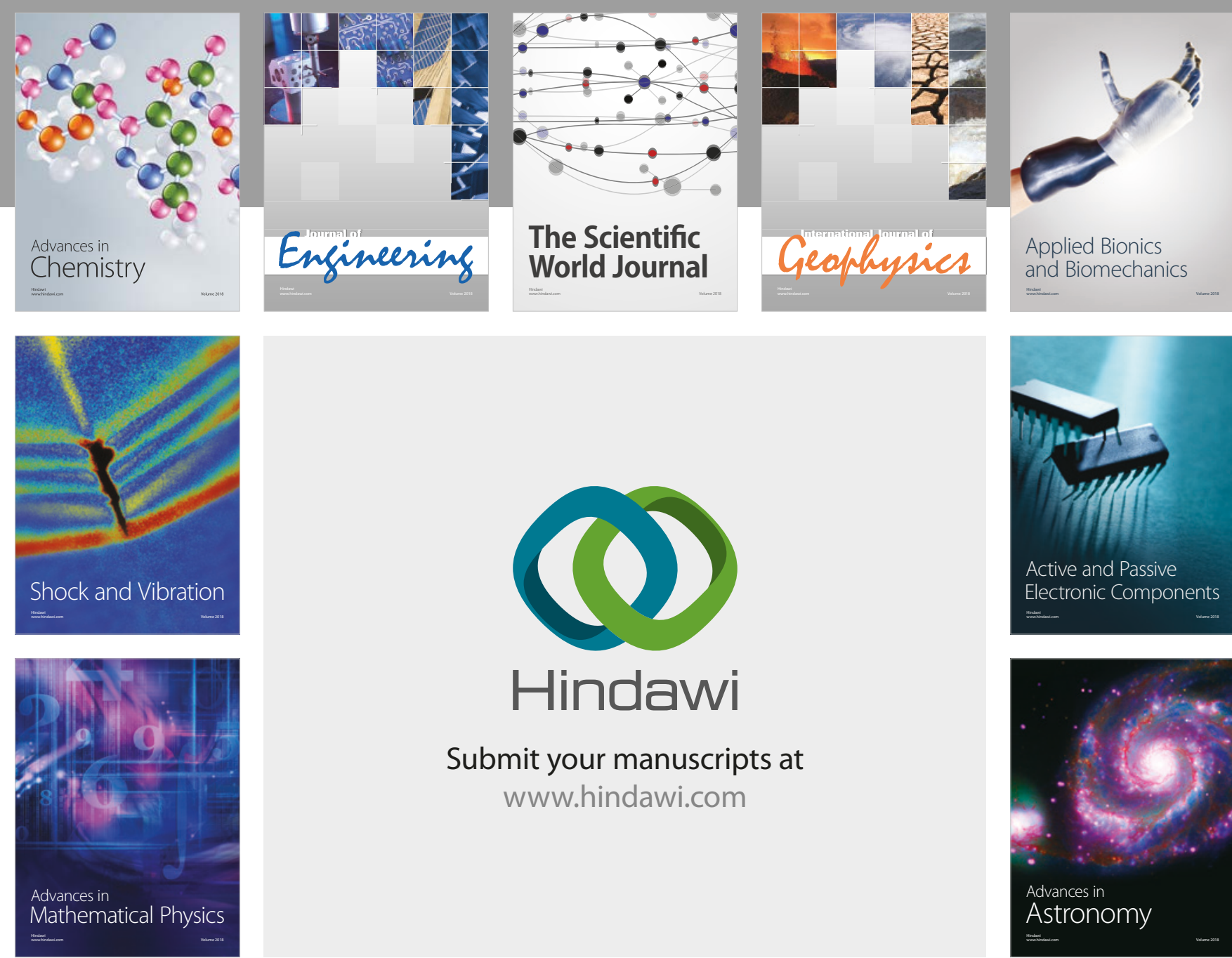

Submit your manuscripts at

www.hindawi.com

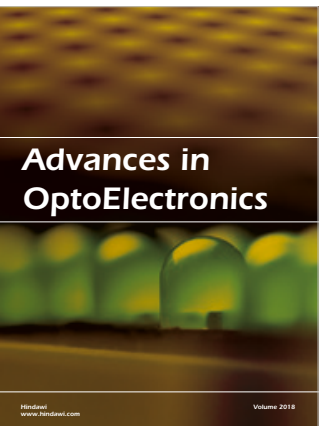

\section{Rotcting Machinery}
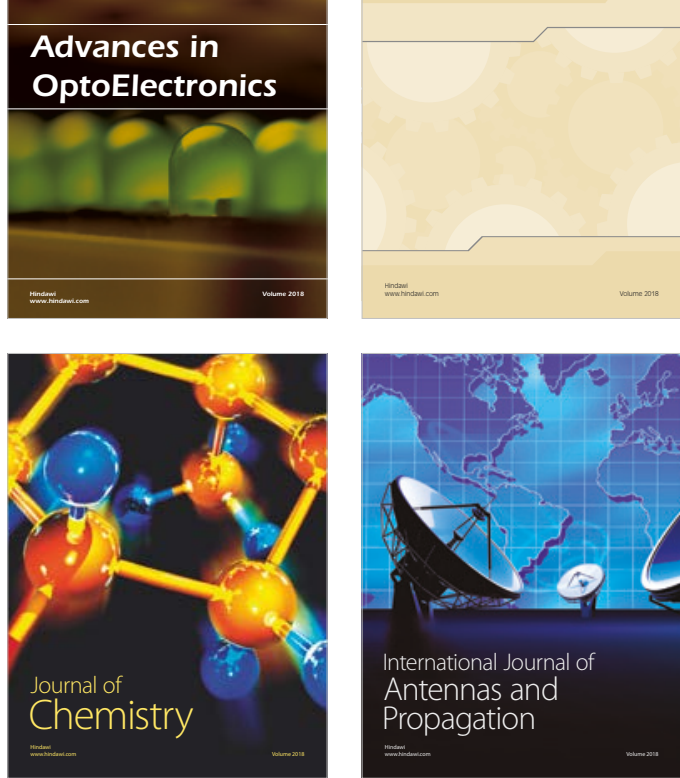

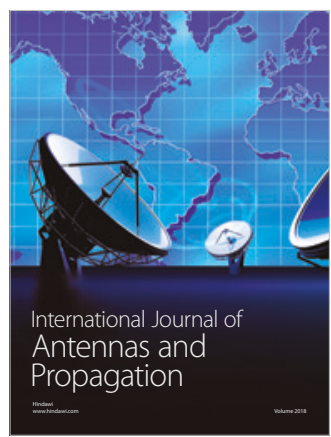

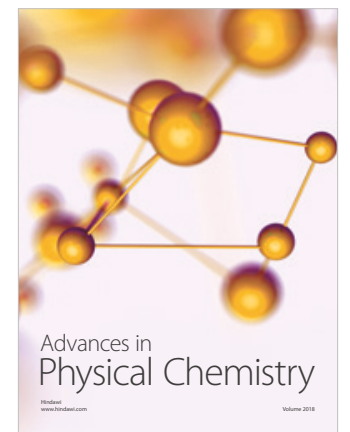

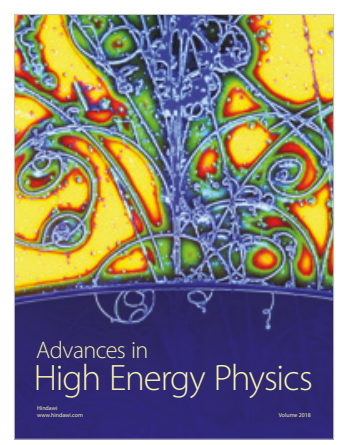

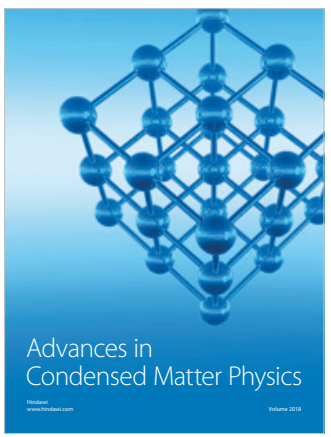

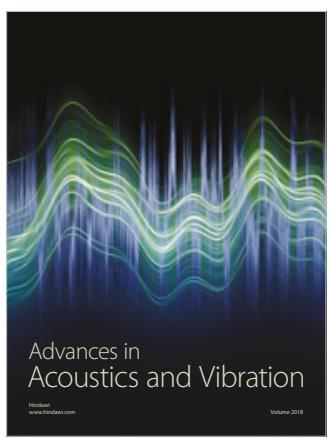

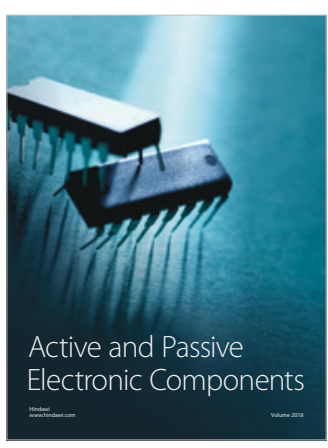
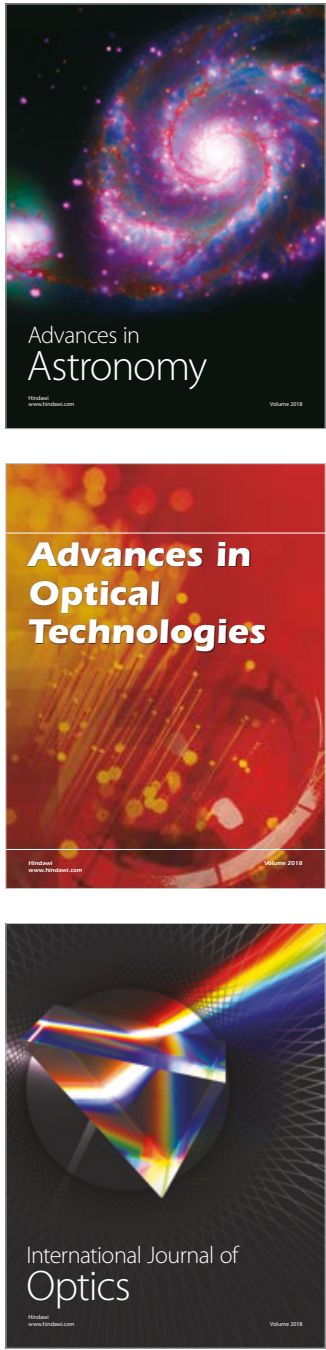